\title{
Supervised and Unsupervised Neural Network Methods applied to Textile Quality Control based on Improved Wavelet Feature Extraction Techniques.
}

\author{
D.A. Karras ${ }^{1}$, S.A. Karkanis ${ }^{2}$ and B.G. Mertzios ${ }^{3}$ \\ ${ }^{1}$ University of Ioannina, Department of Informatics, Ioannina 45110, Greece, dakarras@ cs.uoi.gr \\ ${ }^{2}$ NRCPS “Democritos”, Inst. of Nuclear Technology, Aghia Paraskevi, 15310 Athens, Greece, stavros@zeus.int-rpnet.ariadne-t.gr \\ ${ }^{3}$ Democritus Univ.of Thrace, Dept. of Electr.and Comp. Eng., 67100 Xanthi, Greece, mertzios@demokritos.cc.duth.gr
}

\begin{abstract}
This paper aims at investigating novel solutions to the problem of textile defect detection from images, that can find applications in building robust quality control vision based systems in textile production. The proposed solutions focus on detecting defects from the textural properties of their corresponding wavelet transformed images. More specifically a novel methodology is investigated for discriminating defects in textile images by applying supervised and unsupervised neural classification techniques, employing multilayer perceptrons (MLP) - trained with the on-line backpropagation algorithm - and Kohonen's Self-Organizing Feature Maps (SOFM) respectively. These parallel techniques are applied to innovative wavelet based feature vectors. These vectors are extracted from the wavelet transformed original images using the cooccurrence matrices framework and SVD analysis. The results of the proposed methodology are illustrated in defective textile images where the defective areas are recognized with about $98.5 \%$ accuracy.
\end{abstract}

Keywords : Neural Networks, Wavelets, Texture, Cooccuurence matrices, Quality Control

\section{Introduction}

Defect recognition from images is becoming increasingly significant in a variety of applications since quality control plays a very important role in the manufacturing of virtually every product. Despite the lot of interest, relatively little work has been done in this field since this classification problem presents many difficulties. More specifically the defective areas in quality control images can be viewed as abnormalities or abrupt changes in their statistical properties with varying spatial probability distributions not only within the set of images but also within a single image space. Moreover, the existence of variations in image statistical properties which could be characterized as normal renders defect detection an even more difficult task. Also, the lack of unique characteristics concerning geometry etc. explains more completely the difficulties encountered. However, the resurgence of interest for neural network research has revealed the existence of powerful classifiers which do not depend on the specific probability distribution characterizing their input space. Moreover, these parallel techniques have exhibited improved performance in approximating the 
probability distribution underlying the input data compared to other conventional non-parametric regression techniques. Among these parallel algorithms, MLP plays the most important role in supervised probability approximation while Kohonen's SOFM is the best tool for unsupervised probability approximation. Both techniques are invoked in this paper to solve the defect detection problem. We should mention, however, that if parallel unsupervised classification schemes are able to provide acceptable solutions they are probably the solution of choice, since the multitude of textile defect cases renders supervised training a less practical approach. On the other hand, the emergence of the 2-D wavelet transform [5],[6] as a popular tool in image processing offers the ability of robust feature extraction from images. Combinations of both neural network and wavelet techniques have been used with success in various applications [10]. Therefore, it is worth attempting to investigate whether they can jointly offer a viable solution to the defect recognition problem. To this end, we propose a novel methodology in detecting defective areas in images by examining the discrimination abilities of their textural properties in the wavelet domain. Besides neural network classifiers and the 2-D wavelet transform, the tools utilized in such an analysis are cooccurrence matrix based textural feature extraction [4] and SVD analysis.

The problem of defect detection in the literature is usually considered as deciding about the presence or absence of defects in the original images. However, it is much more useful to consider it as the approximation of the defect spatial probability distribution within the original image since then, production line problems could automatically be detected and probably fixed. This is the approach adopted in this paper which significantly differs from the conventional ones.

Therefore, the problem at hand can be clearly viewed as an image segmentation one, where the image should be segmented in defective and non defective areas only unlike its conventional consideration. Concerning the classical segmentation problem, that is dividing an image into homogeneous regions, the discovery of a generally effective scheme remains a challenge. To this end, many interesting techniques have been suggested so far including spatial frequency techniques and relevant ones like texture clustering in the wavelet domain [9]. Most of these methodologies use very simple features like the energy of the wavelet channels or the variance of the wavelet coefficients [3, 9].

Our approach stems from this line of research. However, there is need for much more sophisticated feature extraction methods if one wants to solve the segmentation problem in its defect recognition incarnation, taking into account the high accuracy required in defect description. Following this reasoning we propose to incorporate in the research efforts the cooccurrence matrices analysis, since it offers a very accurate tool for describing image characteristics and especially texture [4]. It clearly provides second order information about pixel intensities when the majority of the other feature extraction techniques do not exploit it at all. In addition, SVD analysis can localize 
important information concerning the pixel intensity matrices, which has recently been demonstrated that it can effectively be used in invariant pattern recognition [8]. Two are the main stages of the suggested system. Namely, enhanced feature selection in the wavelet domain (enhanced in terms of the information these features carry) and neural network based classification. The viability of the concepts and methods employed in the proposed approach is illustrated in the experimental section of the paper, where it is clearly shown that, by achieving a nearly $98.50 \%$ defective area classification accuracy, with either the supervised or unsupervised parallel classification methods involved, our methodology is very promising for use in the quality control field.

\section{The Wavelet Transformation}

Wavelets offer a general mathematical approach for hierarchical function decomposition. According to this transformation, a function, which can be a function representing an image, a curve, signal etc., can be described in terms of a coarse level in addition with details that range from broad to narrow scales. Wavelets offer an novel technique for computing the levels of detail present, under a framework that is based on a chain of approximation vector spaces $\left\{\mathrm{V}_{j} \subset L^{2}\left(\Re^{2}\right), j \in \mathrm{Z}\right\}$ and a scaling function $\phi$ such that the set of functions $\left\{2^{-j / 2} \phi\left(2^{-j} t-k\right): k \in \mathrm{Z}\right\}$ forming an orthonormal basis for $\mathrm{V}_{j}$. These two components introduce a mathematical framework presented by Mallat [11] and called multiresolution analysis.

A multiresolution analysis (MRA) scheme of $L^{2}\left(\Re^{2}\right)$ can be defined as a sequence of closed subspaces $\left\{\mathrm{V}_{j} \subset L^{2}\left(\Re^{2}\right), j \in \mathrm{Z}\right\}$ satisfying the following properties :

- Containment : $\quad V_{j} \subset V_{j-1} \subset L^{2}$; for all $j \in \mathrm{Z}$.

- Decrease : $\quad \lim _{j \rightarrow \infty} V_{j}=0$, i.e. $\bigcap_{j>N} V_{j}=\varnothing$, for all $\mathrm{N}$.

- Increase : $\quad \lim _{j \rightarrow-\infty} V_{j}=L^{2}$, i.e. $\bigcup_{j<N} V_{j}=L^{2}$, for all $\mathrm{N}$.

- Dilation: $\quad u(2 t) \in V_{(j-1)} \Leftrightarrow u(t) \in V_{j}$.

- Generator: There is a function $\phi \in V_{0}$ whose translation $\{\phi(t-k: k \in \mathrm{Z})\}$ forms a basis for $V_{0}$

By defining complementary subspaces $W_{j}=V_{j-1}-V_{j}$ so that $V_{j-1}=V_{j}+W_{j}$ then we can write, according to the "increase" property that

$$
L^{2}\left(\Re^{2}\right)=\sum_{j \in Z} W_{j}
$$


The subspaces $W_{j}$ are called wavelet subspaces and contain the difference in signal information between the two spaces $V_{j}$ and $V_{j-1}$. These sets contribute to a wavelet decomposition of $L^{2}$ according to (1). In [11] has been proved that a mother wavelet $\psi$ can be created such that the set of functions $\left\{\psi\left(2^{-j} t-k\right): k \in \mathrm{Z}\right\}$ forms a basis for $W_{j}$. The spaces $W_{j}$ are mutually orthogonal and the set of scaled and dilated wavelets $\left\{2^{-j / 2} \psi\left(2^{-j} t-k\right): j \in \mathrm{Z}, k \in \mathrm{Z}\right\}$ provide an orthonormal wavelet basis for $L^{2}\left(\Re^{2}\right)$. Approximating and detailed signals can be obtained by projecting the input signal to the corresponding (approximation or detailed) space. Practically the approximation and detail projection coefficients associated with $V_{j}$ and $W_{j}$ are computed from the approximation and detail coefficients at the next higher scale $V_{j-1}$, using a Quadrature Mirror Filter (QMF) pair and a pyramidal subband coding scheme [12,13].

\section{Improved Feature Extraction in the Wavelet Domain}

The problem of defective area segmentation in textile images using texture discrimination or other second order information, is considered in the wavelet domain, since it has been demonstrated that the discrete wavelet transform (DWT) can lead to better texture modeling [1]. Also, in this way we can better exploit the well known local information extraction properties of wavelet signal decomposition as well as the well known features of wavelet denoising procedures [7]. We use the popular 2-D discrete wavelet transform scheme ([5],[6] etc.) in order to obtain the wavelet analysis of the original images containing defects. It is expected that the images considered in the wavelet domain should be smooth but due to the well known time-frequency localization properties of the wavelet transform, the defective areas- whose statistics vary from the ones of the image backgroundshould more or less clearly emerge from the background. We have experimented with the standard 2D Wavelet transform using nearly all the well known wavelet bases like Haar, Daubechies, Coiflet, Symmlet etc. as well as with Meyer's and Kolaczyk's 2-D Wavelet transforms [6]. However, and this is very interesting, only the 2-D Haar wavelet transform has exhibited the expected and desired properties. All the other orthonormal, continuous and compactly supported wavelet bases have smoothed the images so much that the defective areas don't appear in the subbands. We have performed a one-level wavelet decomposition of the images, thus resulting in four main wavelet channels. Among the three channels 2, 3, 4 (frequency index) we have selected for further processing the one whose histogram presents the maximum variance. A lot of experimentation has shown that this is the channel corresponding to the most clear appearance of the defective areas. The subsequent step in the proposed methodology is to raster scan the image obtained from the selected wavelet channel with sliding windows of M x M dimensions. We have experimented with 256 x 256 images 
and we have found that $\mathrm{M}=8$ is a good size for the sliding window. For each such window we perform two types of analysis in order to obtain improved features in terms of information content.

First, we use the information that comes from the cooccurrence matrices [4]. These matrices represent the spatial distribution and the dependence of the gray levels within a local area. Each $(i, j)$ th entry of the matrices, represents the probability of going from one pixel with gray level (i) to another with a gray level (j) under a predefined distance and angle. More matrices are formed for specific spatial distances and predefined angles. From these matrices, sets of statistical measures are computed (called feature vectors) for building different texture models. We have considered four angles, namely $0^{\circ}, 45^{\circ}, 90^{\circ}, 135^{\circ}$ as well as a predefined distance of one pixel in the formation of the cooccurrence matrices. Therefore, we have formed four cooccurrence matrices. Due to computational complexity issues regarding cooccurrence matrices analysis we have quantized the image obtained from the selected wavelet channel into 16 gray levels instead of the usual 256 levels, without diverse effects in defective area recognition accuracy. This procedure, also, renders the online implementation of the proposed system highly feasible. Among the 14 statistical measures, originally proposed by Haralick [4], that are derived from each cooccurrence matrix we have considered only four of them. Namely, angular second moment, correlation, inverse difference moment and entropy.

- Energy - Angular Second Moment

$$
\begin{gathered}
\boldsymbol{f}_{\boldsymbol{l}}=\sum_{i} \sum_{j} p(i, j)^{2} \\
\boldsymbol{f}_{2}=\frac{\sum_{i=1}^{N_{g}} \sum_{j=1}^{N_{s}}(i * j) p(i, j)-\mu_{*} \mu_{v}}{\sigma_{*} \sigma_{y}} \\
\boldsymbol{f}_{3}=\sum_{i} \sum_{j} \frac{1}{1+(i-j)} p(i, j) \\
\boldsymbol{f}_{4}=-\sum_{i} \sum_{j} p(i, j) \log (p(i, j))
\end{gathered}
$$$$
\text { - Inverse Difference Moment }
$$$$
\text { - Entropy }
$$

These measures, we have experimentally found, that provide high discrimination accuracy that can be only marginally increased by adding more measures in the feature vector. Thus, using the above mentioned four cooccurrence matrices we have obtained 16 features describing spatial distribution within each $8 \times 8$ sliding window in the wavelet domain. In addition, we have formed another set of 8 features for each such window by extracting the singular values of the matrix corresponding to this window. SVD analysis has recently been successfully related to invariant pattern recognition [8]. Therefore, it is reasonable to expect that it provides a meaningful means for characterizing each sliding window, thus preserving first order information regarding this window, while, on the other hand, the cooccurence matrices analysis extracts second order information. 
Therefore, we have formed, for each sliding window, a feature vector containing 24 features that uniquely characterizes it. These feature vectors feed the supervised and unsupervised parallel classification methods of the subsequent stage of the suggested methodology, next described.

\section{Supervised and Unsupervised segmentation of defective areas.}

After obtaining information about the first and second order characteristics of the wavelet transformed images, utilizing the above depicted methodology, we employ supervised and unsupervised neural network architectures to solve the segmentation problem already defined. More specifically, a supervised neural network architecture of the multilayer feedforward type (MLPs), trained with the online backpropagation error algorithm is used, having as goal to decide whether a region belongs to a defective part or not. Also, a Kohonen's SOFM model is utilized aimed at clustering and labeling the pixels of an original image as belonging to defective areas or not.

The inputs to the MLP network are the 24 features of the feature vector extracted from each sliding window. The best network architecture that has been tested in our experiments is the 24-3535-1. This network has been trained with the on line BP algorithm having learning rate equal to 0.3 and momentum coefficient equal to 0.4. The desired outputs during training are determined by the corresponding sliding window location. More specifically, if a sliding window belongs to a defective area the desired output of the network is one, otherwise it is zero. We have defined, during MLP training phase, that a sliding window belongs to a defective area if any of the pixels in the $4 \times 4$ central window inside the original $8 \times 8$ corresponding sliding window belongs to the defect. The reasoning underlying this definition is that the decision about whether a window belongs to a defective area or not should come from a large neighborhood information, thus preserving the 2-D structure of the problem and not from information associated with only one pixel (e.g the central pixel). In addition and probably more significantly, by defining the two classes in such a way, we can obtain many more training patterns for the class corresponding to the defective area, since defects, normally, cover only a small area of the original image. It is important for the effective neural network classifier learning to have enough training patterns for each one of the two classes but, on the other hand, to preserve as much as possible the a priori spatial probability distribution of the problem. We have experimentally found that a proportion of 1:3 for the training patterns belonging to defective and non-defective areas respectively, is a good compromise for achieving both goals.

The same 24 features are the inputs to the SOFM network. The SOFM model used is a $30 \times 20$ two-dimensional map with rectangular neighborhood of gaussian type. The training and test patterns applied to the SOFM were the same feature vectors extracted from the sliding windows previously described in the MLP case. The reference vectors of the map were initialized using small random 
values. The running length of the ordering phase of the map training, has been set to 2000, while its corresponding initial learning rate and initial neighborhood radius have been set to 0.05 and 25 respectively. Finally, the running length of the convergence phase of the map training, has been set to 15000 , while its corresponding initial learning rate and initial neighborhood radius have been set to 0.015 and 5 respectively.

\section{Results and Discussion.}

The efficiency of our approach in recognizing defects in automated inspection textile images, based on utilizing wavelet texture information, is illustrated in the two textile images shown in fig. 1 The first one, which contains a very thin and long defect in its upper side as well as some smaller defects elsewhere, has been used to train the supervised and unsupervised parallel classification methods involved. This image is 256 x 256, while the four wavelet channels obtained by applying the 2-D Haar wavelet transform are 128 x 128. These wavelet channels are shown in fig. 2. In fig. 3 the selected wavelet channel 3 of maximum histogram variance is shown. There exist 14641 sliding windows of $8 \times 8$ size in this wavelet channel. The supervised and unsupervised neural networks involved have been trained with a training set containing 1009 patterns extracted from these sliding windows as described above. 280 out of the 1009 patterns belong to the long and thin defective area of the upper side only, while the rest belong to the class of non defective areas. The neural networks have been tested on all the 14641 patterns coming from the sliding windows of the maximum histogram variance wavelet channel of the first original image of fig.1. They have also been applied to the 14641 patterns extracted - through the same methodology - from the second original image of fig. 1, which is unknown to them. The results are shown in fig. 4, 5, 6, 7. Note that the networks based on the suggested methodology were able to generalize and find also some other minor defects in the images, while other networks of the same type trained with the 64 pixel values of the sliding windows, under exactly the same conditions, were able to find only the largest defects. This fact demonstrates the efficiency of our feature extraction methodology based on wavelet textural and SVD features. Finally, in terms of classification accuracy we have achieved an overall $98.50 \%$. The evolution of the MLP training error and its generalization ability for the class corresponding to defects is shown in fig. 8, 9 respectively.

\section{Conclusions}

We have proposed novel methodologies for detecting defects in automated inspection textile images based on wavelet and neural network segmentation methods, both supervised and unsupervised, by exploiting information coming from textural analysis and SVD in the wavelet 
channels of the 2-D Haar wavelet transformed original images. The efficiency of this approach is illustrated and the classification accuracy obtained is very high for both supervised and unsupervised parallel classifications methods involved. Clearly, our methodology deserves further evaluation in quality control vision based systems.

\section{References}

[1] Ryan, T. W., Sanders, D., Fisher, H. D. and Iverson, A. E. "Image Compression by Texture Modeling in the Wavelet Domain", IEEE trans. Image Processing, Vol. 5, No. 1, pp. 26-36, 1996.

[2] Antonini, M., Barlaud, M., Mathieu, P. and Daubechies, I. "Image Coding Using Wavelet Transform", IEEE trans. Image Processing, Vol.1, pp. 205-220, 1992.

[3] Unser, M. "Texture Classification and Segmentation Using Wavelet Frames", IEEE trans. Image Processing, Vol. 4, No. 11, pp.1549-1560, 1995

[4] Haralick, R. M., Shanmugam, K. and Dinstein, I. "Textural Features for Image Classification", IEEE Trans. Systems, Man and Cybernetics, Vol. SMC-3, No. 6, pp. 610-621, 1973.

[5] Meyer, Y. "Wavelets: Algorithms and Applications", Philadelphia: SIAM, 1993

[6] Kolaczyk, E. "WVD Solution of Inverse Problems", Doctoral Dissertation, Stanford University, Dept. of Statistics, 1994

[7] Donoho, D. L. and Johnstone, I. M. "Ideal Time-Frequency Denoising." Technical Report, Dept. of Statistics, Stanford University.

[8] Al-Shaykh, O.K. and Doherty, J.E. "Invariant Image Analysis based on Radon Transform and SVD.", IEEE Trans. Circuits and Systems, Feb. 1996, Vol. 43, 2, pp. 123-133.

[9] Porter, R. and Canagarajah, N. "A Robust Automatic Clustering Scheme foe Image Segmentation Using Wavelets", IEEE Trans. on Image Processing, April 1996, Vol. 5, No. 4, pp.662 - 665 .

[10]Lee, C. S., et. al, "Feature Extraction Algorithm based on Adaptive Wavelet Packet for Surface Defect Classification”, presented at the ICIP 96, 16-19 Sept. 1996, Lausanne, Switzerland.

[11] Mallat, S.G., “A theory for multiresolution signal decomposition: The wavelet representation”, IEEE Trans. on Pattern Analysis and Machine Intell., Jul. 1989, Vol. 11, No. 7, pp. 674-693.

[12]Kocur, C.M., Rogers, S.K., et. al . "Using Neural Networks to Select Wavelet Features for Breast Cancer", IEEE Eng. in Medice and Biology, May/June 1996, Vol. 15, No. 3, pp. 95-102. [13] Wickerhauser, M.V., "Adapted Wavelet Analysis from Theory to Software", 1994, IEEE Press. 


\section{List of Figure Captions}

Figure 1. Textile images containing representative defects

Figure 2. 2-D Haar basis wavelet transformation of the textile image 1(a)

Figure 3. QMF Channel No.3 of Image 1(a)

Figure 4. Image 1(a) segmented by the MLP

Figure 5. Image 1(a) segmented by the Kohonen's SOFM

Figure 6. Image 1(b) segmented by the MLP

Figure 7. Image 1(b) segmented by the Kohonen's SOFM

Figure 8. MLP Learning Error Evolution

Figure 9. MLP Generalization Performance Evolution 


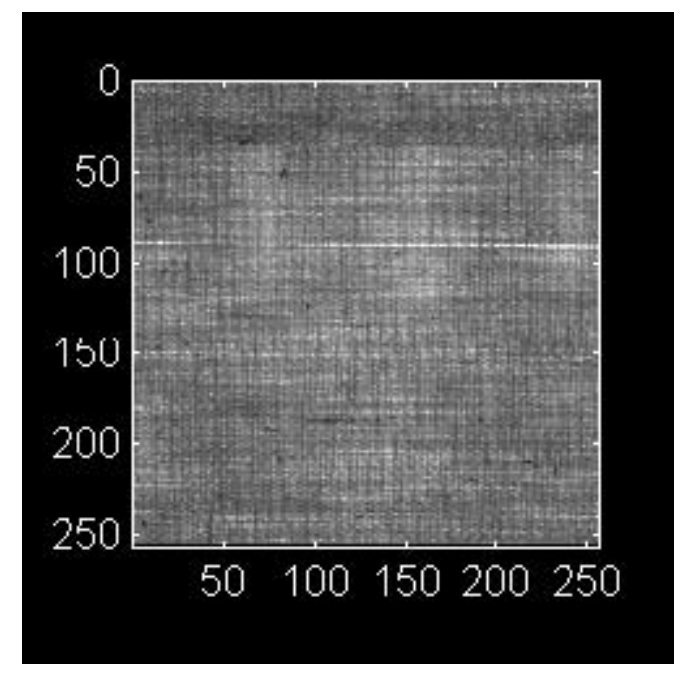

(a)

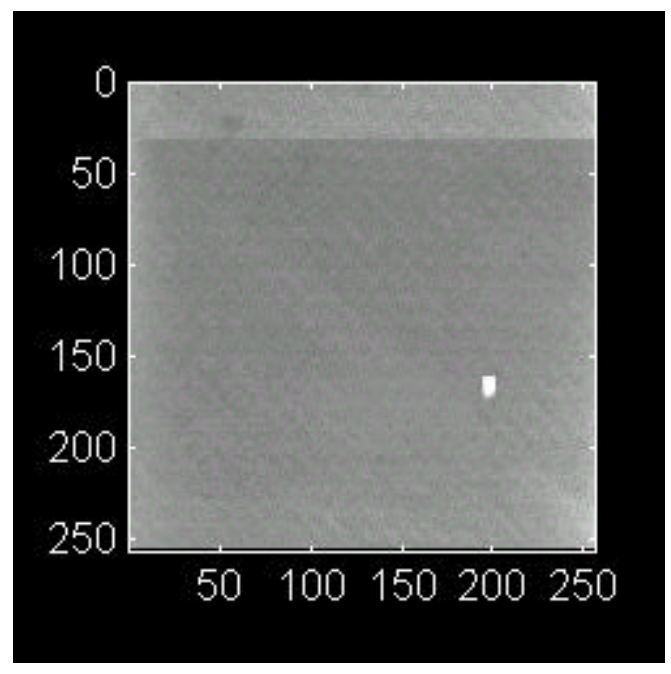

(b)

Figure 1

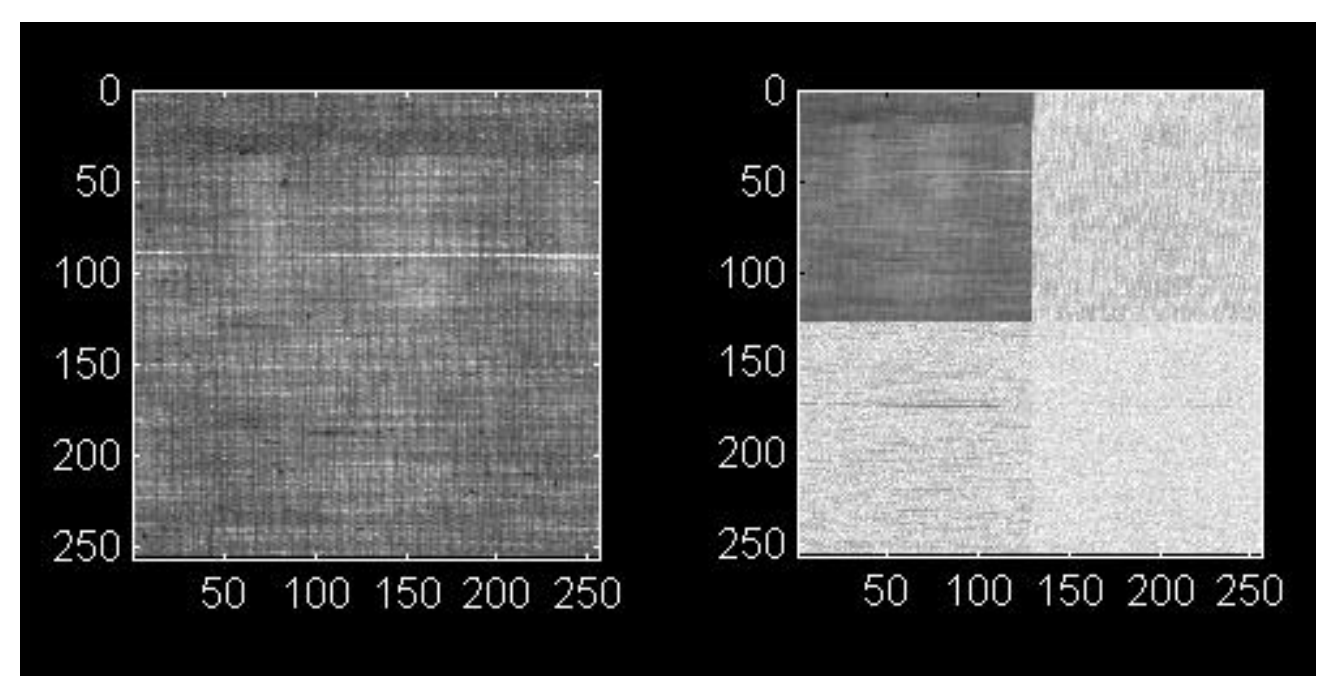

Figure 2

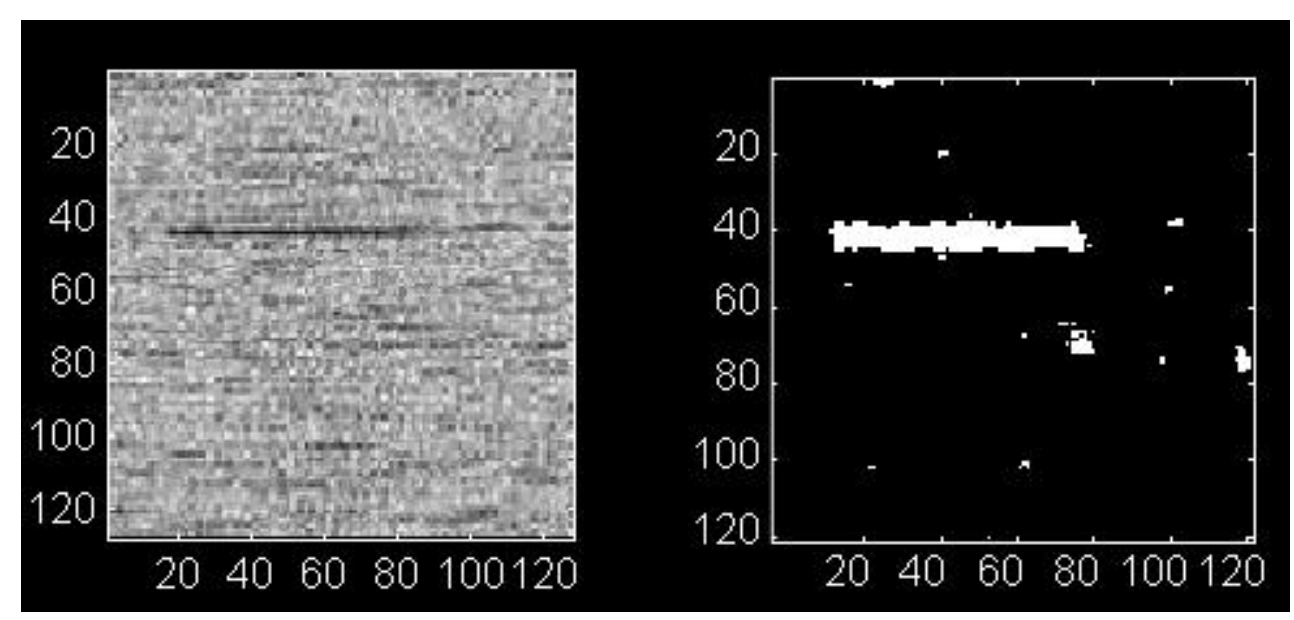

Figure 3

Figure 4 


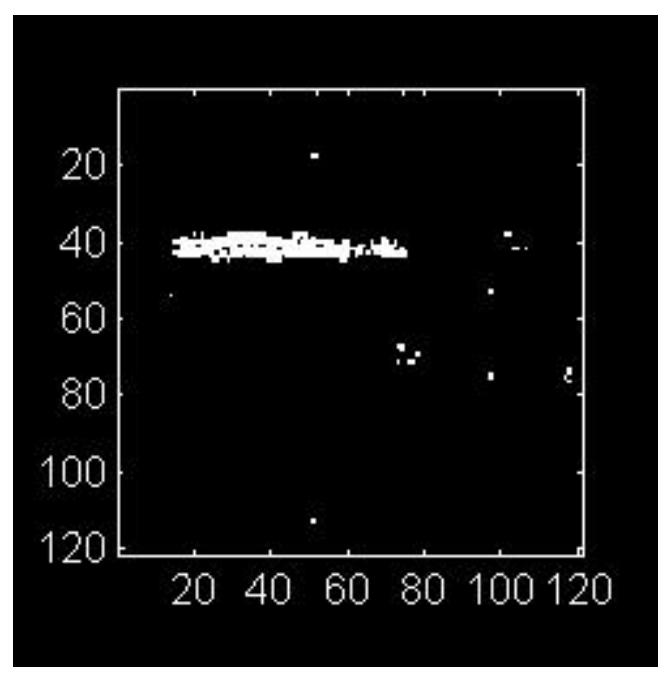

Figure 5

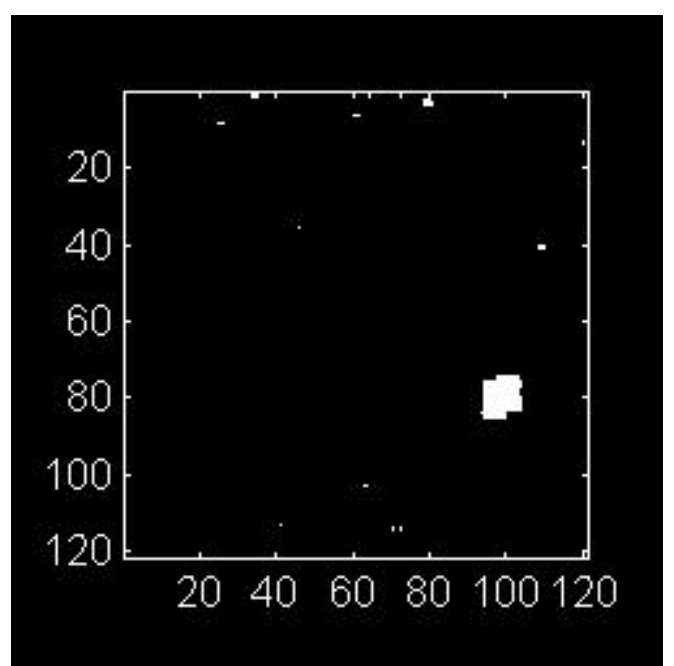

Figure 6

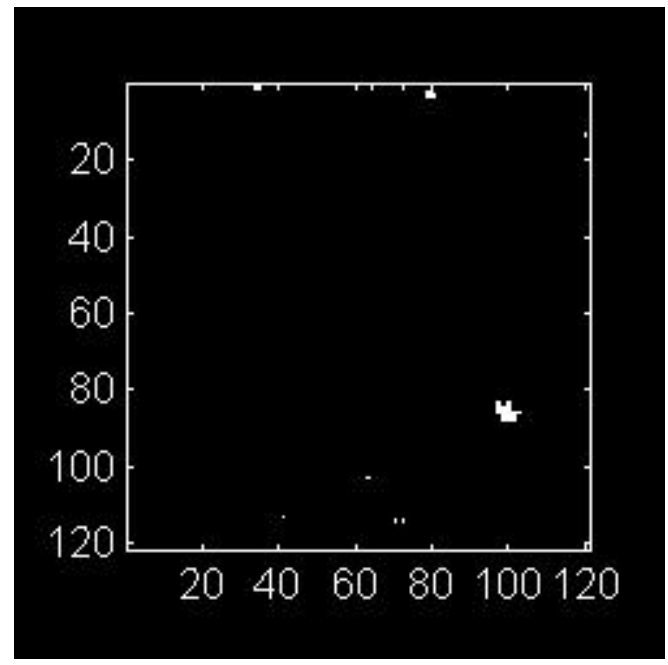

Figure 7

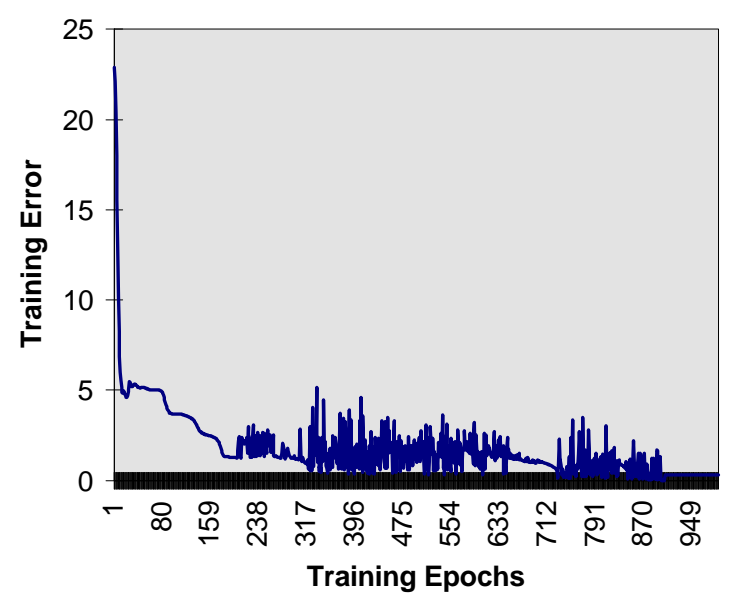

Figure 8

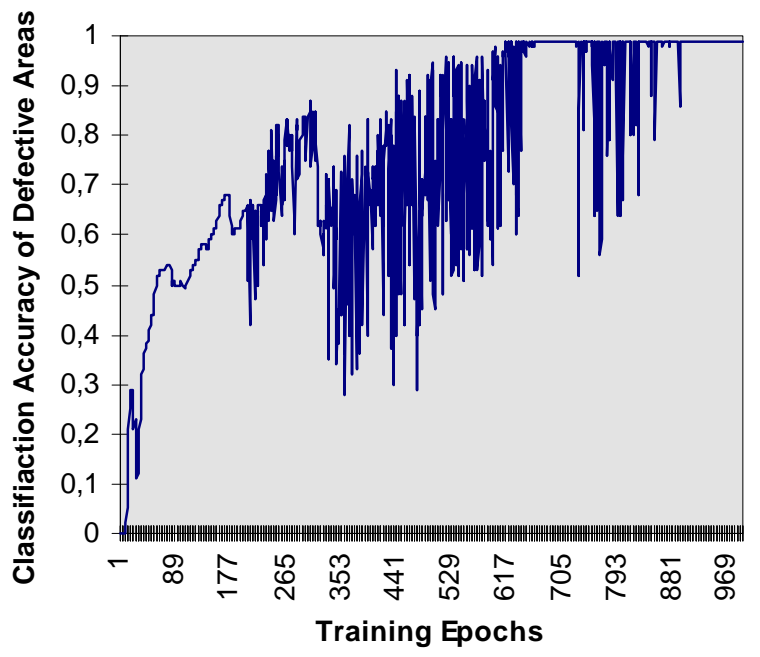

Figure 9 